Article

\title{
Vector Competence of Italian Populations of Culicoides for Some Bluetongue Virus Strains Responsible for Recent Northern African and European Outbreaks
}

\author{
Valentina Federici ${ }^{1, *(D)}$, Maria Goffredo ${ }^{1}$, Giuseppe Mancini ${ }^{1}$, Michela Quaglia ${ }^{1}$, \\ Adriana Santilli ${ }^{1}$, Francesca Di Nicola ${ }^{1}$, Matteo De Ascentis ${ }^{1}$, Pierangela Cabras ${ }^{2}$, \\ Carmela Volpicelli ${ }^{3}$, Claudio De Liberato ${ }^{4}$, Giuseppe Satta ${ }^{2}$, Giovanni Federico ${ }^{5}$ (D), \\ Alessandra Leone ${ }^{1}$, Maura Pisciella ${ }^{1}$, Ottavio Portanti ${ }^{1}{ }^{\mathbb{D}}$, Federica Pizzurro ${ }^{1}$, Liana Teodori ${ }^{1}$ \\ and Giovanni Savini ${ }^{1}$ \\ 1 Istituto Zooprofilattico Sperimentale dell'Abruzzo e del Molise 'G. Caporale', Via Campo Boario, \\ 64100 Teramo, Italy; m.goffredo@izs.it (M.G.); g.mancini@izs.it (G.M.); m.quaglia@izs.it (M.Q.); \\ a.santilli@izs.it (A.S.); f.dinicola@izs.it (F.D.N.); m.deascentis@izs.it (M.D.A.); a.leone@izs.it (A.L.); \\ m.pisciella@izs.it (M.P.); o.portanti@izs.it (O.P.); f.pizzurro@izs.it (F.P.); 1.teodori@izs.it (L.T.); \\ g.savini@izs.it (G.S.) \\ 2 Istituto Zooprofilattico Sperimentale della Sardegna, Via Duca degli Abruzzi 8, 07100 Sassari, Italy; \\ pierangela.cabras@izs-sardegna.it (P.C.); giuseppe.satta@izs-sardegna.it (G.S.) \\ 3 Azienda Sanitaria Provinciale Crotone, Via M. Nicoletta, 88900 Crotone (KR), Italy; \\ veterinari.areaa@asp.crotone.it \\ 4 Istituto Zooprofilattico Sperimentale del Lazio e della Toscana, Via Appia Nuova 1411, 00178 Rome, Italy; \\ claudio.deliberato@izslt.it \\ 5 Istituto Zooprofilattico Sperimentale del Mezzogiorno, Via Figurella, 89135 Catona (RC), Italy; \\ giovanni.federico@izsmportici.it \\ * Correspondence: v.federici@izs.it; Tel.: +39-0861-332416-406
}

Received: 30 August 2019; Accepted: 9 October 2019; Published: 12 October 2019

\begin{abstract}
The distribution of Bluetongue virus (BTV) in Europe can be represented by two distinct and interconnected epidemiological systems (episystems), each characterized by different ecological characteristics and vector species. This study investigated the vector competence of Italian populations of Culicoides imicola and Culicoides obsoletus/scoticus to some representative BTV strains after artificial oral infection. The BTV strains were selected according to their ability to spread to one or both episystems and included BTV-4 ITA, responsible of the recent Italian and French BTV-4 outbreaks; the BTV-2 strain which caused the first BTV incursion in Italy, Corsica, and Balearic Islands; BTV-4 MOR, responsible for the epidemic in Morocco; and BTV-8, the strain which spread through Europe between 2006 and 2008. Blood-soaked cotton pledgets and Hemotek membrane feeder using Parafilm ${ }^{\circledR}$ membrane were used to artificially feed midges. For each population/strain, recovery rates (positive/tested heads) were evaluated using serogroup- and serotype-specific RT-PCR. The trial demonstrated that, except for the Abruzzo population of C. obsoletus/C. scoticus, which was refractory to BTV-4 MOR infection, all the investigated Culicoides populations are susceptible to the selected BTV strains and that, if prompt vaccination programs and restriction measures had not been implemented, BTV-2 and BTV-4 MOR could have spread all over Europe.
\end{abstract}

Keywords: Culicoides imicola; Culicoides obsoletus; Culicoides scoticus; bluetongue virus; oral infection; vector competence; recovery rate; Italy 


\section{Introduction}

Culicoides biting midges (Diptera: Ceratopogonidae) are biological vectors of several important arboviruses such as those causing Bluetongue (BT), African horse sickness, and Epizootic hemorrhagic disease. Because of the direct effect of the disease on animals, but especially because of the ensuing ban on international trade of ruminants and their products between BTV-infected and non-infected areas, outbreaks of Bluetongue virus (BTV) continue to have significant economic impacts in Europe and worldwide. In relation to the spread of BTV strains, two distinct and interconnected epidemiological systems (episystems), with different ecological characteristics and vector species, have been identified in Europe. The concept of episystem includes the set of biological, environmental, and epidemiological elements of a disease in defined geographic and temporal scales [1]. For BT, it is known that distinct strains of BTV (virus topotypes) vectored by different species of Culicoides occur in specific regions of the world. The topotypes of BTV and the vector species that occur within each episystem are relatively stable, despite extensive and ongoing trade and movement of ruminants between individual episystems.

One episystem is distributed in Southern Europe (Mediterranean Basin), where BTV is primarily spread by Culicoides imicola, and one in Southern, Central, and Northern Europe, in which species of the Obsoletus complex act as main vectors [2-4]. In the recent European incursions, some BTV strains were able to successfully spread and adapt in both episystems, while some other strains remained confined to one episystem only. It has been hypothesized that this capacity to spread and adapt to different episystems is somehow related to the vector competence of the Culicoides species present. It has been observed that the BTV spreading in the US western states was strictly associated to the different distribution of the two more common indigenous species, Culicoides sonorensis and Culicoides variipennis, and to their different vector competence [5]. Due to its particular geographic position, Italy comprises both episystems. Culicoides imicola and species of the Obsoletus complex are geographically complementary and together cover the whole Italian peninsula [6].

The Obsoletus complex is a monophyletic group. In Italy, it is composed almost exclusively by two species, Culicoides obsoletus and Culicoides scoticus, although a third species, Culicoides montanus, is rarely recorded. This study aimed to investigate the vector competence of different Italian populations of Culicoides imicola and C. obsoletus/scoticus to some BTV strains responsible for recent incursions in the Mediterranean basin and Europe after artificial oral feeding. Four BTV strains (BTV-4 ITA 2014, BTV-2 2001, BTV-4 MOR, BTV-8 2006) were selected based on their ability to spread to one or two episystems.

In Italy, BTV appeared for the first time in Sardinia in 2000. This incursion, due to BTV-2, caused severe clinical signs and death in the affected sheep. BTV-2 then rapidly spread to Sicily and to other regions of South-Central Italy (Basilicata, Lazio, Campania, and Tuscany) [7]. Even if the most implicated vector of the Sardinian BTV-2 outbreaks was C. imicola, other Culicoides species, including C. obsoletus and C. scoticus, contributed to the BTV spread in Italy [8]. BTV-2 was in fact isolated from species of the Obsoletus complex caught in Campania and Apulia regions [9]. BTV-2 has never been reported in Central-Northern Europe. Since this first occurrence, several other BTV incursions were reported in the Italian territory, involving serotypes 1, 2, 4, 9, 16, 8, and 3 [10,11]. The second BTV strain selected for this study was the BTV-8 responsible for the most severe BT epidemic ever reported in Europe. After emerging in Central Europe in 2006, it further spread to Northern and Southern European countries. It appeared in Italy in 2008, invading Northern Italy and the Sardinia region in 2009. The third selected BTV strain was the BTV-4, originating from the Balkan peninsula, which has been responsible for the recent Italian (2014-2019) and French (2018-2019) epidemics. This strain has been detected in pools of the Obsoletus complex (Apulia) [3] and in pools of C. imicola in Sardinia in 2017 (unpublished data). Finally, the fourth selected strain was a BTV-4 reassortant strain, containing sequences from previous BTV-1 (MOR2006/06) and BTV-4 (MOR 2004/02) strains that spread in Morocco and neighboring regions. It circulated in Morocco in 2009 [12] but was never reported in Italy or in Central-Northern Europe. With a positive correlation between disease and its abundance/distribution, C. imicola was suggested to be the main vector species [13]. 
The Culicoides populations included in this study were selected based on episystems and presence/ abundance of indigenous species: C. imicola of the Sardinia and Calabria regions was selected as representative of the Southern Europe episystem and C. obsoletus/scoticus of the Abruzzo and Lazio regions as representative of the other episystem.

In this study, two artificial blood feeding methods were used: the Hemotek membrane system and cotton wool pledgets. Because of their reluctance to feed under laboratory conditions, few artificial oral feeding and BTV vector competence studies have been carried out on species of the Obsoletus complex [14-18]. By contrast, as it is relatively easy to feed C. imicola through a membrane and via cotton wool pledgets [19], the susceptibility of C. imicola to a variety of BTV strains has been frequently determined in the lab [20-24]. In this study, the feeding rate and the vector competence for both taxa were investigated.

\section{Materials and Methods}

\subsection{Collection Sites}

Collections of Culicoides were made in four Italian regions (Abruzzo, Lazio, Sardinia, and Calabria) at the selected farms (Figure 1). The suitable collection sites were selected based on Culicoides abundance results derived from the entomological National surveillance plan implemented in Italy since 2000. The insect collections were performed by the team of the Istituto Zooprofilattico Sperimentale dell'Abruzzo e del Molise "G. Caporale" (IZSAM), in collaboration with the network of the Italian Istituti Zooprofilattici Sperimentali (IIZZSS) and the local Veterinary Services.

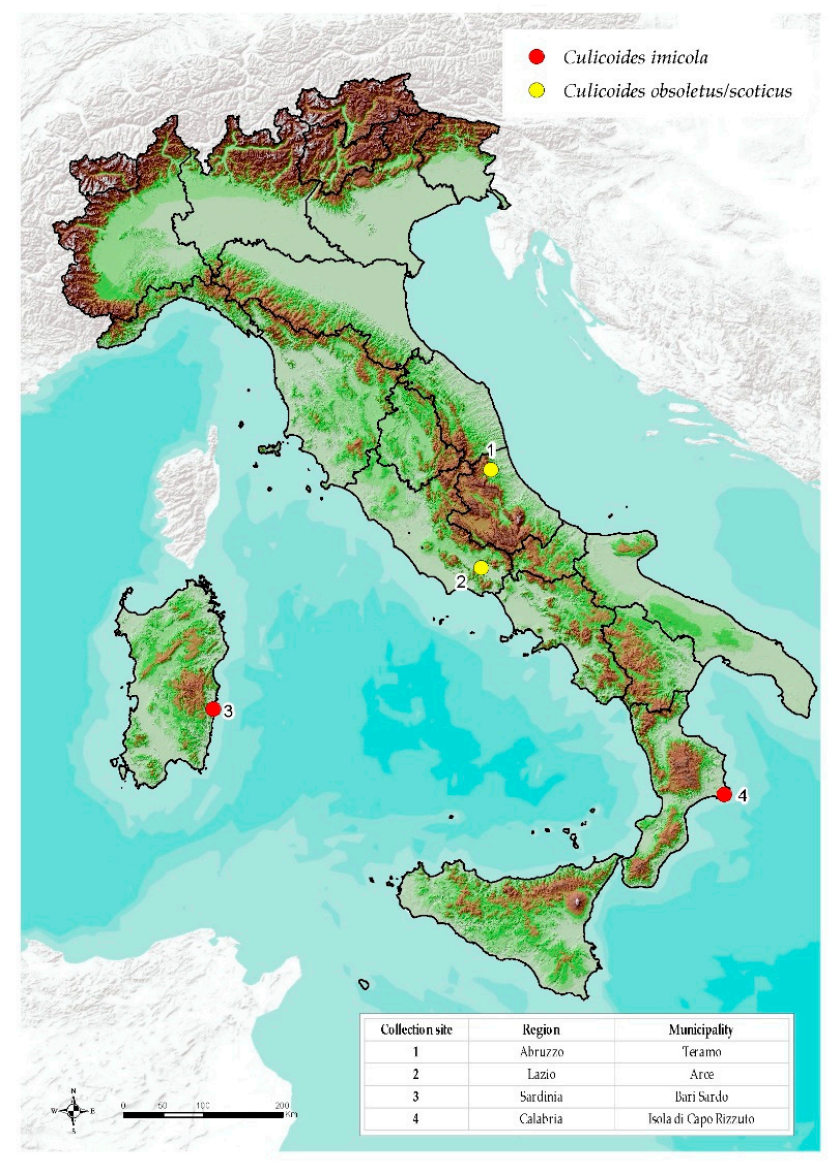

Figure 1. Location of the Italian population of Culicoides obsoletus/scoticus (Abruzzo and Lazio regions) and Culicoides imicola (Sardinia and Calabria regions). 


\subsection{Field Activities}

On each farm, a variable number (4-7) of UV blacklight suction traps operated all night long from about one hour before dusk on two or three consecutive days. Field Culicoides were collected early in the morning by placing the collection beakers in boxes and allowing trapped midges to fly in card glasses through funnels.

Overall, 31 and 30 adult collection nights were performed, from 7 June 2016 to 19 October 2016 and from 20 June 2017 to 18 October 2017, respectively.

Collected Culicoides were then transferred in $64 \mathrm{~mm}$ card boxes and fed with sucrose solution $10 \%$. After two days of acclimatization at $25^{\circ} \mathrm{C}$ and $80 \%>\mathrm{HR}>40 \%$, they were starved for one day before the oral infection.

\subsection{Laboratory Activities}

\subsubsection{Virus Isolates}

For the experimental infection, four BTV strains were selected:

- BTV-2, strain 2000TE8341: It was isolated from the spleen of a Sardinian sheep that died because of BTV in 2000. The strain was passed once onto the Kc cell line and three times onto VERO cells (from African green monkey kidney) before use.

- $\quad$ BTV-4 ITA, strain 2014TE31172: It was isolated from the blood of a sheep infected with BTV in the Apulia region in 2014. The strain was passed once onto the Kc cell line and three times onto VERO cells before use.

- $\quad$ BTV-4 MOR, strain MOR2009-07: Kindly provided by the Pirbright Institute (Lara Harrup), it was isolated from a blood sample of a BTV-infected sheep in Morocco in 2009. This strain was passed three times onto the Kc cell line and twice onto VERO cells before use.

- $\quad$ BTV-8, strain UKG2007-82: Kindly provided by the Pirbright Institute (Lara Harrup), it was isolated from a blood sample of a bovine infected in the UK in 2007. The strain was passed three times onto the Kc cell line and twice onto VERO cells before use.

\subsubsection{Culicoides Oral Infection}

All experimental infection phases were performed at IZSAM in a dedicated room within the BSL3 facilities. To obtain the blood meals for infecting Culicoides, BTV suspensions were diluted (1:3, 1:4 or 1:5) in defibrinated cattle or sheep blood. Culicoides were fed for $45 \mathrm{~min}$ using either the Hemotek blood feeding system with a Parafilm ${ }^{\circledR}$ membrane or the cotton pledgets in accordance with the method described by Venter and colleagues [19]. The Parafilm Hemotek system has already been successfully used for feeding field-collected Culicoides and vector competence studies, obtaining feeding rates comparable with those achieved when using one-day old-chick stretched skins [25-27]. Each feeder unit of the Hemotek system was set to warm the blood meal to $37^{\circ} \mathrm{C}$. A total of 73 feedings were carried out. After each meal, an aliquot of the blood used for feeding was retained and the BTV titer assessed using the Reed and Muench formula [28]. Fed Culicoides were immobilized on dry ice, and fully engorged females of $C$. imicola and C. obsoletus/scoticus were sorted and counted using a refrigerate chill-table and stereomicroscope. Six to thirty-five blooded midges were assayed after feeding ( 0 days post-infection, dpi) to establish baseline infection rates and to determine virus load in each midge. The remainder were incubated in cardboard boxes at $25^{\circ} \mathrm{C}$ and $80 \%>\mathrm{HR}>40 \%$ for 10 days with ad libitum access to $10 \%$ sucrose solution. Those Culicoides which survived the incubation period were killed on dry ice and dissected. The heads and thorax/abdomens of each insect were stored separately at $-80^{\circ} \mathrm{C}$ until further analyses. 


\subsubsection{Species Identification}

Identification keys, mainly based on wing morphology, and a multiplex PCR, based on internal transcribed spacer 2 ribosomal DNA sequences (ITS2), were used to identify midges [4,29-33]. The morphological identification was performed under the stereomicroscope on a chill table according to Delécolle, Campbell and Pelham-Clinton, and Goffredo and Meiswinkel [29-31]. Unlike C. imicola, molecular techniques are required to identify $C$. obsoletus and C. scoticus at species level $[4,32,33]$. From each midge head homogenate, total nucleic acids were extracted using the Biosprint ${ }^{\circledR} 96$ (Qiagen, Hilden, Germany). DNA was used for midge identification while RNA for viral analysis. When species identification was inconclusive, DNA extraction was repeated with the automated Maxwell 16 system (Promega, Madison, WI, USA) with the DNA IQ Casework Sample kit (Promega, Madison, WI, USA) according to manufacturer's instructions. The ITS-2 segment of ribosomal DNA was amplified using the primers 5.8 SF, 28 SR, Scoticus-194R, MOU-316F, and Montanus-227R [32]. In this study, we refer to "C. obsoletus/scoticus" or to "C. obsoletus and C. scoticus" depending on whether they have been identified by morphological or morphological/molecular tools, respectively. Once identified, midges were individually stored at $-80^{\circ} \mathrm{C}$ for virus detection.

\subsubsection{Virus Detection}

The RT-PCR described by Hofmann et al. [34] was used to detect BTV in midge heads. The VetMAX European BTV Typing Kit (Thermo Fisher Scientific, Waltham, MA, USA) was instead used to determine the BTV serotype. The serogroup-specific RT-PCR [34] used in this study is the test recommended by the Office International des Epizooties (OIE). Its performance in the laboratory has been verified and validated for all known BTV serotypes and most of the strains circulating in the Mediterranean Basin according to the ISO 17025 validation guidelines. To control the entire process, Armored RNA West Nile Virus (HNY1999) (Asuragen, Austin, TX, USA) was included in each PCR reaction as an internal positive control. The threshold cycle value used as a cut off for best discriminating between positive and negative samples was 50 . Ct values less than 50 were considered as positive, while those with $\mathrm{Ct}$ values equal to 50 as negative. With regard to the Typing kit, according to the manufacturer's instructions, the $\mathrm{Ct}$ value for the best discrimination between positive and negative samples was 40. For each population/strain, the recovery rate (number of BTV positive heads/number of tested heads) was calculated using both the serogroup- and the serotype-specific RT-PCR. The inclusion of the serotype-specific RT-PCR in this trial was necessary since in Sardinia, on the farm where $C$. imicola midges were trapped for BTV-8 competence studies, BTV-4 was circulating at the time of collection. Being a highly specific test, the typing kit has been used to rule out natural infection with BTV-4; therefore, its results were considered when comparing rates involving BTV-8 from the Sardinian population. On the other hand, results obtained with the serogroup-specific RT-PCR, which is more sensitive than the type-specific RT-PCR, were used in most of the recovery rate comparisons made in this study. In analyzing the recovery rate data, more or less conservative approaches were evaluated based on the RT-PCRs cut off levels. The mean $\mathrm{Ct}$ values detected immediately after feeding $(0 \mathrm{dpi})$ were also compared with those achieved after the incubation period (10 dpi), and any reduction was interpreted as further evidence of virus replication in the salivary glands [25]. In this study, as in other studies [17], midge heads were used to detect the virus and determine the recovery rates. Though unlikely, there was a chance that salivary glands remained in the thorax when heads were separated. However, since salivary gland barriers have not been described for Culicoides species [35], presence of BTV in the head, expressing the complete virus dissemination in the midge, can still be considered a valid indicator of BTV vector competency [17].

\subsection{Statistical Analysis}

Statistical differences on recovery and feeding rates were investigated through a Bayesian approach, using the Beta distribution. Beta distribution is a type of probability distribution. This distribution 
represents a family of probabilities and is a versatile way to represent outcomes for percentages or proportions [36]. A 95\% confidence interval (C.I.) was calculated for each parameter through the Beta distribution. The comparisons between the recovery and feeding rates were considered significant when the confidence intervals, calculated through the beta distribution, did not overlap.

\section{Results}

\subsection{Feeding Rate}

More than 250,000 Culicoides were orally fed in 73 attempts. A total of 6013 midges were engorged after feeding (2203 by Hemotek membrane, 3810 using the cotton method). Of these, 262 were analyzed at $0 \mathrm{dpi}$ and 3218 at $10 \mathrm{dpi}$. Feeding rates and surviving midges at $10 \mathrm{dpi}$ are reported in Table 1 . They were grouped according to collection site, BTV strain, and feeding method. When the Hemotek membrane feeding system was used, not all the Culicoides populations investigated were fed successfully. Due to the very low feeding rates obtained, the use of this system was limited to the Culicoides populations of Sardinia, Abruzzo, and Calabria and to some BTV strains only (Table 1). The feeding method influenced the Culicoides blood-feeding rates, with the cotton pledget $(15.88 \%$, $95 \% \mathrm{CI}=15.43-16.35 \%)$ resulting in a significantly higher $(p<0.05)$ blood-feeding rate than the Hemotek Parafilm ${ }^{\circledR}$ membrane $(0.97 \%, 95 \% \mathrm{CI}=0.93-1.01 \%)$. All feeding attempts made using the cotton pledget method were successful, with rates ranging from $9.6 \%$ for C. imicola (Sardinia BTV-8) to $41.62 \%$ for C. obsoletus/scoticus of Lazio (BTV-8) (Table 1). With regard to the Culicoides species, the $C$. imicola population feeding success rates $(1.02 \%, 95 \% \mathrm{CI}=0.98-1.07 \%)$ were significantly higher $(p<0.05)$ than those of $C$. obsoletus/scoticus $(0.07 \%, 95 \% \mathrm{CI}=0.04-0.13 \%)$ when the Hemotek system was used. Conversely, when cotton pledget was used, a significantly higher rate $(p<0.05)$ of C. obsoletus/scoticus $(16.78 \%, 95 \% \mathrm{CI}=16.11-17.48 \%)$ was successfully fed compared to the $C$. imicola $(15.06 \%, 95 \% \mathrm{CI}=14.45-15.70 \%)$.

\subsection{Competence Studies}

In this study, the virus titer of the blood meals ranged from $10^{5} \mathrm{TCID}_{50} / \mathrm{mL}$ ( $50 \%$ Tissue Culture Infective Dose) to $10^{6.54} \mathrm{TCID}_{50} / \mathrm{mL}$ (Table 2). Of the 262 midges tested immediately after feeding, 225 $(85.88 \%)$ tested positive for BTV (Table 3). Although in most cases, the differences were not significant, the number of positive midges detected using serogroup-specific RT-PCR were generally higher than those detected by serotype-specific RT-PCR (Tables 3 and 4). The Sardinian population of C. imicola fed via the Hemotek system achieved the best recovery rates regardless of BTV strains. The BTV recovery rate resulting from the serotype-specific RT-PCR at $10 \mathrm{dpi}$ from the Sardinian population of C. imicola $(66.39 \%, 95 \% \mathrm{CI}=62.87-69.74 \%)$ fed through the Hemotek membrane was significantly higher than the rate obtained when the same population was fed on cotton wool $(3.60 \%, 95 \% \mathrm{CI}=2.40-5.39 \%)$ (Figure 2; Table 4). The recovery rates remained significantly higher when the more conservative approach, which considered cases where RT-PCR Ct values decreased after the 10-day incubation period, was taken $(48.41 \%, 95 \% \mathrm{CI}=44.78-52.05 \%$ vs. $2.78 \%, 95 \% \mathrm{CI}=1.75-4.41 \%)$ (Tables 5 and 6 ). 
Table 1. Feeding rate and number of surviving midges at 10 days post-infection (dpi) according to strain, feeding method, and population.

\begin{tabular}{|c|c|c|c|c|c|c|c|c|c|}
\hline \multicolumn{2}{|l|}{ BTV-4 ITA } & \multicolumn{4}{|c|}{ Fed via Hemotek } & \multicolumn{4}{|c|}{ Fed via Cotton Pledget } \\
\hline SPECIES & SITE & ENGORGED & TOTAL NUMBER & FEEDING RATE (\%) & $10 \mathrm{dpi}$ & ENGORGED & TOTAL NUMBER & FEEDING RATE (\%) & $10 \mathrm{dpi}$ \\
\hline Culicoides obsoletus/scoticus & Abruzzo & 5 & 9139 & 0.05 & 4 & 566 & 3923 & 14.43 & 190 \\
\hline Culicoides obsoletus/scoticus & Lazio & & & & & 229 & 752 & 30.45 & 186 \\
\hline Culicoides imicola & Sardinia & 1107 & 54,471 & 2.03 & 359 & 330 & 2078 & 15.88 & 258 \\
\hline Culicoides imicola & Calabria & 0 & 2586 & 0 & 0 & 400 & 2196 & 18.21 & 269 \\
\hline \multicolumn{2}{|l|}{ BTV-2 } & \multicolumn{4}{|c|}{ Fed via Hemotek } & \multicolumn{4}{|c|}{ Fed via Cotton Pledget } \\
\hline SPECIES & SITE & ENGORGED & TOTAL NUMBER & FEEDING RATE (\%) & $10 \mathrm{dpi}$ & ENGORGED & TOTAL NUMBER & FEEDING RATE (\%) & $10 \mathrm{dpi}$ \\
\hline Culicoides obsoletus/scoticus & Abruzzo & 4 & 4131 & 0.1 & 1 & 447 & 4589 & 9.74 & 226 \\
\hline Culicoides obsoletus/scoticus & Lazio & & & & & 173 & 516 & 33.53 & 75 \\
\hline Culicoides imicola & Sardinia & 719 & 71,843 & 1 & 257 & 351 & 3376 & 10.40 & 228 \\
\hline Culicoides imicola & Calabria & 0 & 1213 & 0 & 0 & 337 & 2426 & 13.89 & 219 \\
\hline \multicolumn{2}{|l|}{ BTV-8 } & \multicolumn{4}{|c|}{ Fed via Hemotek } & \multicolumn{4}{|c|}{ Fed via Cotton Pledget } \\
\hline SPECIES & SITE & ENGORGED & TOTAL NUMBER & FEEDING RATE (\%) & $10 \mathrm{dpi}$ & ENGORGED & TOTAL NUMBER & FEEDING RATE (\%) & $10 \mathrm{dpi}$ \\
\hline Culicoides obsoletus/scoticus & Abruzzo & & & & & 174 & 484 & 35.95 & 110 \\
\hline Culicoides obsoletus/scoticus & Lazio & & & & & 77 & 185 & 41.62 & 61 \\
\hline Culicoides imicola & Sardinia & 190 & 57,318 & 0.33 & 107 & 153 & 1593 & 9.6 & 125 \\
\hline Culicoides imicola & Calabria & & & & & 165 & 402 & 41.04 & 117 \\
\hline \multicolumn{2}{|l|}{ BTV-4 MOR } & \multicolumn{4}{|c|}{ Fed via Hemotek } & \multicolumn{4}{|c|}{ Fed via Cotton Pledget } \\
\hline SPECIES & SITE & ENGORGED & TOTAL NUMBER & FEEDING RATE (\%) & $10 \mathrm{dpi}$ & ENGORGED & TOTAL NUMBER & FEEDING RATE (\%) & $10 \mathrm{dpi}$ \\
\hline Culicoides obsoletus/scoticus & Abruzzo & & & & & 168 & 720 & 23.33 & 126 \\
\hline Culicoides obsoletus/scoticus & Lazio & & & & & 87 & 278 & 31.29 & 64 \\
\hline Culicoides imicola & Sardinia & 178 & 26,863 & 0.66 & 120 & & & & \\
\hline Culicoides imicola & Calabria & & & & & 153 & 471 & 32.48 & 116 \\
\hline Culicoides obsoletus/scoticus & SUBTOTAL & 9 & 13,270 & 0.07 & 5 & 1921 & 11,447 & 16.78 & 1038 \\
\hline Culicoides imicola & SUBTOTAL & 2194 & 214,294 & 1.02 & 843 & 1889 & 12,542 & 15.06 & 1332 \\
\hline TOTAL & & 2203 & 227,564 & 0.97 & 848 & 3810 & 23,989 & 15.88 & 2370 \\
\hline
\end{tabular}


Table 2. Titers of bluetongue virus (BTV) strains in blood meals.

\begin{tabular}{cc}
\hline Virus & $\begin{array}{c}\text { Minimum-Maximum Virus Titer TCID } \\
\text { 50 } \mathbf{~ m L} \\
\text { (Number of Blood Meals) }\end{array}$ \\
\hline BTV-4 ITA & $10^{5.3}-10^{6.54}(20)$ \\
BTV-2 & $10^{5.01}-10^{6.14}(22)$ \\
BTV-8 & $10^{5}-10^{6.3}(16)$ \\
BTV-4 MOR & $10^{5.68}-10^{6.39}(15)$ \\
\hline
\end{tabular}

With the Hemotek feeding system, the recovery rates of BTV-4 ITA $(70.5 \%$; $95 \%$ CI $=65.6-75 \%)$ and BTV-2 $(66.5 \%, 95 \% \mathrm{CI}=60.6-72 \%)$, obtained at $10 \mathrm{dpi}$ from Sardinian midges were similar $(p>0.05)$. Both rates, however, were higher $(p<0.05)$ than that of BTV-4 MOR $(49.2 \%, 95 \% \mathrm{CI}=40.4-58 \%)$. The recovery rate of BTV-4 ITA was also higher $(p<0.05)$ than that of BTV-8 $(52.3 \%, 95 \% \mathrm{CI}=42.9-61.6 \%)$ (Table 5). If the more conservative approach was used, the recovery rates of BTV-4 ITA, BTV-2, and BTV-8 were similar $(p>0.05)$ but significantly higher $(p<0.05)$ than those of BTV-4 MOR (Tables 5 and 6$)$.

When midges fed on cotton wool pledgets soaked in infected blood, all Culicoides populations tested were susceptible to the BTV strains selected for this trial, with the exception of the C. obsoletus/scoticus population of Abruzzo and BTV-4 MOR. However, the BTV recovery rates obtained from C. imicola were significantly higher than those from C. obsoletus/scoticus $(0.83 \%, 95 \% \mathrm{CI}=0.41-1.69 \% ; 0.35 \%$, $95 \% C I=0.13-1.03 \%$; ) when either the serotype-specific RT-PCR $(2.55 \%, 95 \% \mathrm{CI}=1.81-3.60 \%)$ or the $0-10$ dpi Ct value reduction $(1.64 \%, 95 \% \mathrm{CI}=1.07-2.53 \%)$ were used as criteria for determining the vector competence. If considered individually, the recovery rate of BTV-2 from the C. obsoletus/scoticus population of Lazio $(12 \% ; 95 \% \mathrm{CI}=6.5-21.3 \%)$ was significantly $(p<0.05)$ higher than those from the Abruzzo $(2.7 \% ; 95 \% \mathrm{CI}=1.2-5.7 \%)$ and Calabria $(3.2 \% ; 95 \% \mathrm{CI}=1.6-6.4 \%)$ midge populations. Similarly, the BTV-4 MOR recovery rate from the Abruzzo Culicoides obsoletus/scoticus population $(0 \% ; 95 \% \mathrm{CI}=0-2.3 \%)$ was significantly lower than those from C. obsoletus/scoticus of Lazio (6.3\%; $95 \% \mathrm{CI}=2.5-15 \%)$ and Culicoides imicola of Calabria $(6.9 \% ; 95 \% \mathrm{CI}=3.6-13 \%)$ (Tables 4 and 6$)$. These differences disappeared ( $p>0.05 \%$ ) when the 0-10 dpi $\mathrm{Ct}$ value reductions were used to determine the vector susceptibility to different BTV-strain infection (Table 5). This more conservative approach, however, revealed a significant higher recovery rate of BTV-4 ITA from the C. imicola population of Sardinia compared to those determined for C. obsoletus/scoticus of Abruzzo.

With regard to C. obsoletus/scoticus populations either from Lazio (66.2\%) or Abruzzo (77.2\%), the number of $C$. scoticus collected and identified was higher than that of $C$. obsoletus. With the exception of BTV-2 from the Abruzzo population of C. obsoletus and BTV-8 from the Lazio population of C. scoticus, all BTV strains examined in this study were recovered from both species with similar rates $(p>0.05)$ (Table 7). 
Table 3. Virus detection in Culicoides tested immediately after feeding on BTV-infected blood at 0 days post-infection.

\begin{tabular}{|c|c|c|c|c|c|c|c|c|c|}
\hline \multirow[b]{2}{*}{ Feeding Method } & \multirow[b]{2}{*}{ BTV Strain } & \multirow[b]{2}{*}{ Population } & \multirow[b]{2}{*}{ Number of Tested } & \multicolumn{3}{|c|}{ Serogroup-Specific RT PCR (Positive Midges $\mathrm{Ct}<50$ ) } & \multicolumn{3}{|c|}{ Serotype-Specific RT PCR (Positive Midges Ct $<40$ ) } \\
\hline & & & & Number of Positive & Recovery Rate (\%) & Ct Mean (Min-Max) & Number of Positive & Recovery Rate (\%) & Ct Mean (Min-Max) \\
\hline cotton & 4 ITA & C. obsoletus/scoticus Abruzzo & 16 & 14 & 87.5 & $36(31-44)$ & l & I & 1 \\
\hline cotton & 4 ITA & C. obsoletus/scoticus Lazio & 11 & 11 & 100 & $38(36-39)$ & 11 & 100 & $33(32-35)$ \\
\hline Hemotek & 4 ITA & C. imicola Sardinia & 35 & 33 & 94.3 & $37(35-42)$ & 33 & 94.3 & $33(30-36)$ \\
\hline cotton & 4 ITA & C. imicola Sardinia & 10 & 8 & 80 & $40(38-42)$ & 8 & 80 & $35(33-37)$ \\
\hline cotton & 4 ITA & C. imicola Calabria & 15 & 15 & 100 & $39(36-42)$ & 15 & 100 & $33(31-36)$ \\
\hline cotton & 2 & C. obsoletus/scoticus Abruzzo & 15 & 12 & 80 & $37(33-42)$ & 1 & 6.7 & $39(39-39)$ \\
\hline cotton & 2 & C. obsoletus/scoticus Lazio & 11 & 11 & 100 & $38(36-39)$ & 11 & 100 & $34(33-35)$ \\
\hline Hemotek & 2 & C. imicola Sardinia & 21 & 19 & 90.5 & $39(36-43)$ & 18 & 85.7 & $34(32-39)$ \\
\hline cotton & 2 & C. imicola Sardinia & 14 & 7 & 50 & $43(41-44)$ & 4 & 28.6 & $38(36-39)$ \\
\hline cotton & 2 & C. imicola Calabria & 12 & 12 & 100 & $40(35-43)$ & 12 & 100 & $35(32-38)$ \\
\hline cotton & 8 & C. obsoletus/scoticus Abruzzo & 17 & 12 & 70.6 & $42(39-47)$ & 7 & 41.2 & $35(34-36)$ \\
\hline cotton & 8 & C. obsoletus//scoticus Lazio & 7 & 4 & 57.1 & $41(39-42)$ & 2 & 28.6 & $36(34-37)$ \\
\hline Hemotek & 8 & C. imicola Sardinia & 9 & 1 & I & 1 & 8 & 88.9 & $35(32-37)$ \\
\hline cotton & 8 & C. imicola Sardinia & 13 & 1 & 1 & 1 & 9 & 69.2 & $36(35-37)$ \\
\hline cotton & 8 & C. imicola Calabria & 15 & 13 & 86.7 & $39(37-41)$ & 13 & 86.7 & $35(33-37)$ \\
\hline cotton & $4 \mathrm{MOR}$ & C. obsoletus/scoticus Abruzzo & 13 & 10 & 76.9 & $42(38-49)$ & 10 & 76.9 & $35(33-38)$ \\
\hline cotton & $4 \mathrm{MOR}$ & C. obsoletus/scoticus Lazio & 8 & 7 & 87.5 & $40(37-47)$ & 7 & 87.5 & $33(31-35)$ \\
\hline Hemotek & $4 \mathrm{MOR}$ & C. imicola Sardinia & 6 & 6 & 100 & $36(34-38)$ & 6 & 100 & $31(29-32)$ \\
\hline cotton & $4 \mathrm{MOR}$ & C. imicola Calabria & 14 & 14 & 100 & $39(36-41)$ & 13 & 92.9 & $34(31-37)$ \\
\hline
\end{tabular}

Table 4. Virus detection in Culicoides (heads) fed on BTV-infected blood at 10 days post-infection.

\begin{tabular}{|c|c|c|c|c|c|c|c|}
\hline \multirow[b]{2}{*}{ Feeding Method } & \multirow[b]{2}{*}{ BTV Strain } & \multirow[b]{2}{*}{ Population } & \multirow[b]{2}{*}{ Number of Tested } & \multicolumn{2}{|c|}{ Serogroup-Specific RT PCR (Positive Midges Ct $<50$ ) } & \multicolumn{2}{|c|}{ Serotype-Specific RT PCR (Positive Midges $\mathrm{Ct}<40$} \\
\hline & & & & Number of Positive & Ct Mean (Min-Max) & Number of Positive & Ct Mean (Min-Max) \\
\hline Hemotek & 4 ITA & C. obsoletus/scoticus Abruzzo & 4 & 0 & 1 & 1 & 1 \\
\hline cotton & 4 ITA & C. obsoletus/scoticus Abruzzo & 190 & 9 & $40(34-46)$ & 1 & $29(29-29)$ \\
\hline cotton & 4 ITA & C. obsoletus/scoticus Lazio & 186 & 18 & $41(25-47)$ & 2 & $33(27-39)$ \\
\hline Hemotek & 4 ITA & C. imicola Sardinia & 359 & 275 & $32(24-48)$ & 253 & $30(23-39)$ \\
\hline cotton & 4 ITA & C. imicola Sardinia & 258 & 17 & $36(27-41)$ & 9 & $33(27-38)$ \\
\hline cotton & 4 ITA & C. imicola Calabria & 269 & 15 & $40(26-47)$ & 6 & $33(24-37)$ \\
\hline Hemotek & 2 & C. obsoletus/scoticus Abruzzo & 1 & 0 & 1 & 1 & 1 \\
\hline cotton & 2 & C. obsoletus/scoticus Abruzzo & 226 & 6 & $38(27-42)$ & 1 & $30(30-30)$ \\
\hline cotton & 2 & C. obsoletus/scoticus Lazio & 75 & 9 & $38(33-40)$ & 2 & $36(33-38)$ \\
\hline Hemotek & 2 & C. imicola Sardinia & 257 & 186 & $32(22-47)$ & 171 & $29(23-39)$ \\
\hline cotton & 2 & C. imicola Sardinia & 228 & 16 & $40(32-45)$ & 12 & $35(30-39)$ \\
\hline cotton & 2 & C. imicola Calabria & 219 & 7 & $41(28-45)$ & 2 & $32(25-38)$ \\
\hline
\end{tabular}


Table 4. Cont.

\begin{tabular}{|c|c|c|c|c|c|c|c|}
\hline \multirow[b]{2}{*}{ Feeding Method } & \multirow[b]{2}{*}{ BTV Strain } & \multirow[b]{2}{*}{ Population } & \multirow[b]{2}{*}{ Number of Tested } & \multicolumn{2}{|c|}{ Serogroup-Specific RT PCR (Positive Midges $\mathrm{Ct}<50$ ) } & \multicolumn{2}{|c|}{ Serotype-Specific RT PCR (Positive Midges $\mathrm{Ct}<40$} \\
\hline & & & & Number of Positive & Ct Mean (Min-Max) & Number of Positive & Ct Mean (Min-Max) \\
\hline cotton & 8 & C. obsoletus/scoticus Abruzzo & 110 & 4 & $43(39-46)$ & 1 & $38(38-38)$ \\
\hline cotton & 8 & C. obsoletus/scoticus Lazio & 61 & 2 & $42(39-44)$ & 0 & 1 \\
\hline Hemotek & 8 & C. imicola Sardinia & 107 & l & 1 & 56 & $30(27-38)$ \\
\hline cotton & 8 & C. imicola Sardinia & 125 & i & / & 1 & $30(30-30)$ \\
\hline cotton & 8 & C. imicola Calabria & 117 & 12 & $40(31-44)$ & 1 & $34(34-34)$ \\
\hline cotton & $4 \mathrm{MOR}$ & C. obsoletus/scoticus Abruzzo & 126 & 0 & 1 & 1 & 1 \\
\hline cotton & $4 \mathrm{MOR}$ & C. obsoletus/scoticus Lazio & 64 & 4 & $40(38-43)$ & 0 & 1 \\
\hline Hemotek & $4 \mathrm{MOR}$ & C. imicola Sardinia & 120 & 62 & $38(31-45)$ & 59 & $32(27-38)$ \\
\hline cotton & $4 \mathrm{MOR}$ & C. imicola Calabria & 116 & 8 & $38(33-43)$ & 6 & $32(29-39)$ \\
\hline
\end{tabular}

Table 5. Recovery rates of bluetongue virus strains from different Italian vector populations orally infected according to different RT-PCR cut-off levels.

\begin{tabular}{|c|c|c|c|c|c|c|c|}
\hline \multirow[b]{2}{*}{ Feeding Method } & \multirow[b]{2}{*}{ BTV Strain } & \multirow[b]{2}{*}{ Population } & \multirow[b]{2}{*}{ Number of Tested } & \multicolumn{2}{|c|}{ Serogroup-Specific RT-PCR } & \multicolumn{2}{|c|}{ Serotype-Specific RT-PCR } \\
\hline & & & & $\begin{array}{l}\text { Number of Positive with } \\
\mathrm{Ct}<50 \text { (Recovery Rate\%) }\end{array}$ & $\begin{array}{l}\text { Number of Positive with } \\
\mathrm{Ct}<\text { Mean Ct at } 0 \mathrm{dpi} \\
\text { (Recovery Rate\%) }\end{array}$ & $\begin{array}{l}\text { Number of Positive with } \\
\mathrm{Ct}<40 \text { (Recovery Rate\%) }\end{array}$ & $\begin{array}{l}\text { Number of Positive with } \\
\text { Ct }<\text { Mean Ct at } 0 \mathrm{dpi} \\
\text { (Recovery Rate\%) }\end{array}$ \\
\hline cotton & 4 ITA & C. obsoletus/scoticus Abruzzo & 190 & $9(4.7)$ & $1(0.5)$ & $1(0.5)$ & I \\
\hline cotton & 4 ITA & C. obsoletus/scoticus Lazio & 186 & $18(9.7)$ & $2(1.1)$ & $2(1.1)$ & $1(0.5)$ \\
\hline Hemotek & 4 ITA & C. imicola Sardinia & 359 & $275(76.6)$ & $201(56)$ & $253(70.5)$ & $165(46)$ \\
\hline cotton & 4 ITA & C. imicola Sardinia & 258 & $17(6.6)$ & $13(5)$ & $9(3.5)$ & $6(2.3)$ \\
\hline cotton & 4 ITA & C. imicola Calabria & 269 & $15(5.6)$ & $4(1.5)$ & $6(2.2)$ & $1(0.4)$ \\
\hline cotton & 2 & C. obsoletus/scoticus Abruzzo & 226 & $6(2.7)$ & $2(0.9)$ & $1(0.4)$ & $1(0.4)$ \\
\hline cotton & 2 & C. obsoletus/scoticus Lazio & 75 & $9(12.0)$ & $2(2.7)$ & $2(2.7)$ & $1(1.3)$ \\
\hline Hemotek & 2 & C. imicola Sardinia & 257 & $186(72.4)$ & $148(57.6)$ & $171(66.5)$ & $131(51.4)$ \\
\hline cotton & 2 & C. imicola Sardinia & 228 & $16(7.0)$ & $11(4.8)$ & $12(5.3)$ & $10(4.4)$ \\
\hline cotton & 2 & C. imicola Calabria & 219 & $7(3.2)$ & $2(0.9)$ & $2(0.9)$ & $1(0.5)$ \\
\hline cotton & 8 & C. obsoletus/scoticus Abruzzo & 110 & $4(3.6)$ & $2(1.8)$ & $1(0.9)$ & $0(0)$ \\
\hline cotton & 8 & C. obsoletus/scoticus Lazio & 61 & $2(3.3)$ & $1(1.6)$ & $0(0)$ & $0(0)$ \\
\hline Hemotek & 8 & C. imicola Sardinia & 107 & 1 & 1 & $56(52.3)$ & $54(50.5)$ \\
\hline cotton & 8 & C. imicola Sardinia & 125 & 1 & 1 & $1(0.8)$ & $1(0.8)$ \\
\hline cotton & 8 & C. imicola Calabria & 117 & $12(10.3)$ & $2(1.7)$ & $1(0.9)$ & $1(0.9)$ \\
\hline cotton & $4 \mathrm{MOR}$ & C. obsoletus/scoticus Abruzzo & 126 & $0(0)$ & $0(0)$ & $0(0)$ & $0(0)$ \\
\hline cotton & $4 \mathrm{MOR}$ & C. obsoletus/scoticus Lazio & 64 & $4(6.3)$ & $2(3.1)$ & $0(0)$ & $0(0)$ \\
\hline Hemotek & $4 \mathrm{MOR}$ & C. imicola Sardinia & 120 & $62(51.7)$ & $16(13.3)$ & $59(49.2)$ & $21(17.5)$ \\
\hline cotton & $4 \mathrm{MOR}$ & C. imicola Calabria & 116 & $8(6.9)$ & $5(4.3)$ & $6(5.2)$ & $4(3.4)$ \\
\hline
\end{tabular}


Table 6. Comparison of the recovery rates (serogroup-specific/serotype-specific RT-PCR positive heads/tested heads at 10 days post-infection) from different Italian vector populations, orally infected with BTV strains.

\begin{tabular}{|c|c|c|c|c|c|c|}
\hline \multirow[b]{2}{*}{ RT-PCR } & \multirow[b]{2}{*}{ Population } & \multirow[b]{2}{*}{ Feeding Method } & \multicolumn{4}{|c|}{ Recovery Rate \% (Confidence Intervals \%) } \\
\hline & & & BTV-4 ITA & BTV-2 & BTV-8 & BTV-4 MOR \\
\hline \multirow{5}{*}{ Serogroup-specific RT-PCR } & C. obsoletus/scoticus Abruzzo & Cotton & $\begin{array}{c}0.5 \\
(0.1-2.9)\end{array}$ & $\begin{array}{c}0.9 \\
(0.3-3.1)\end{array}$ & $\begin{array}{c}1.8 \\
(0.6-6.4)\end{array}$ & $\begin{array}{c}0 \\
(0-2.3)\end{array}$ \\
\hline & C. obsoletus/scoticus Lazio & Cotton & $\begin{array}{c}1.1 \\
(0.3-3.8)\end{array}$ & $\begin{array}{c}2.7 \\
(0.8-9.2)\end{array}$ & $\begin{array}{c}1.6 \\
(0.4-8.7)\end{array}$ & $\begin{array}{c}3.1 \\
(1-10.7)\end{array}$ \\
\hline & \multirow{2}{*}{ C. imicola Sardinia } & Hemotek & $\begin{array}{c}56 \\
(50.8-61)\end{array}$ & $\begin{array}{c}57.6 \\
(51.5-63.5)\end{array}$ & / & $\begin{array}{c}13.3 \\
(8.4-20.6)\end{array}$ \\
\hline & & Cotton & $\begin{array}{c}5 \\
(3-8.4) \\
\end{array}$ & $\begin{array}{c}4.8 \\
(2.7-8.4)\end{array}$ & / & / \\
\hline & C. imicola Calabria & Cotton & $\begin{array}{c}1.5 \\
(0.6-3.7)\end{array}$ & $\begin{array}{c}0.9 \\
(0.3-3.2)\end{array}$ & $\begin{array}{c}1.7 \\
(0.5-6)\end{array}$ & $\begin{array}{c}4.3 \\
(1.9-9.7)\end{array}$ \\
\hline \multirow{5}{*}{ Serotype-specific RT-PCR } & C. obsoletus/scoticus Abruzzo & Cotton & / & $\begin{array}{c}0.4 \\
(0.1-2.4)\end{array}$ & $\begin{array}{c}0 \\
(0-2.7)\end{array}$ & $\begin{array}{c}0 \\
(0-2.3)\end{array}$ \\
\hline & C. obsoletus/scoticus Lazio & Cotton & $\begin{array}{c}0.5 \\
(0.1-2.9)\end{array}$ & $\begin{array}{c}1.3 \\
(0.3-7.1)\end{array}$ & $\begin{array}{c}0 \\
(0-4.7)\end{array}$ & $\begin{array}{c}0 \\
(0-4.5)\end{array}$ \\
\hline & \multirow{2}{*}{ C. imicola Sardinia } & Hemotek & $\begin{array}{c}46 \\
(40.9-51.1)\end{array}$ & $\begin{array}{c}51.4 \\
(44.9-57)\end{array}$ & $\begin{array}{c}50.5 \\
(41.1-59.8)\end{array}$ & $\begin{array}{c}17.5 \\
(11.8-25.3)\end{array}$ \\
\hline & & Cotton & $\begin{array}{c}2.3 \\
(1.1-5)\end{array}$ & $\begin{array}{c}4.4 \\
(2.4-7.9)\end{array}$ & $\begin{array}{c}0.8 \\
(0.2-4.3)\end{array}$ & I \\
\hline & C. imicola Calabria & Cotton & $\begin{array}{c}0.4 \\
(0.1-2)\end{array}$ & $\begin{array}{c}0.5 \\
(0.1-2.5)\end{array}$ & $\begin{array}{c}0.9 \\
(0.2-4.6)\end{array}$ & $\begin{array}{c}3.4 \\
(1.4-8.5)\end{array}$ \\
\hline
\end{tabular}




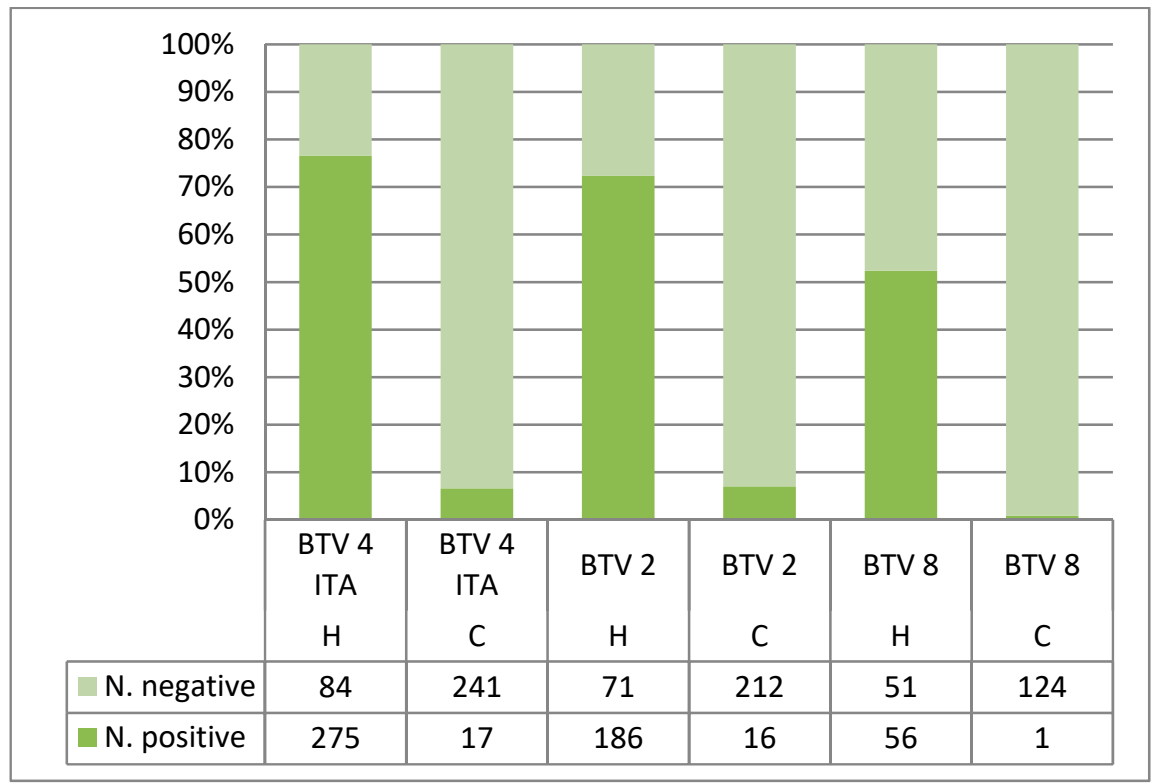

Figure 2. Recovery rate of bluetongue virus strains from Culicoides imicola (Sardinia population) fed through different methods: Hemotek device $(\mathrm{H})$, cotton wool pledgets $(\mathrm{C})$.

Table 7. Recovery rate (serogroup-specific RT-PCR) and relative 95\% confidence interval of Culicoides obsoletus and C. scoticus fed on BTV-infected blood at 0 and 10 days post-infection.

\begin{tabular}{|c|c|c|c|c|c|c|c|}
\hline Dpi & $\begin{array}{l}\text { BTV } \\
\text { Strain }\end{array}$ & Site & Species & $\begin{array}{l}\text { Number of Positive/ } \\
\text { Number of Tested }\end{array}$ & $\begin{array}{c}\text { Recovery } \\
\text { Rate \% }\end{array}$ & $\begin{array}{l}\text { Confidence } \\
\text { Intervals \% }\end{array}$ & $\begin{array}{l}\text { CT Mean } \\
\text { (Min-Max) }\end{array}$ \\
\hline \multirow{18}{*}{0} & \multirow{5}{*}{4 ITA } & \multirow{3}{*}{ Abruzzo } & C. obsoletus & $5 / 6$ & 83.3 & l & $37(32-44)$ \\
\hline & & & C. scoticus & $8 / 9$ & 88.9 & i & $34(31-40)$ \\
\hline & & & C. obsoletus/scoticus * & $1 / 1$ & 100 & l & $41(41-41)$ \\
\hline & & \multirow{2}{*}{ Lazio } & C. obsoletus & $1 / 1$ & 100 & / & 37 (37-37) \\
\hline & & & C. scoticus & $10 / 10$ & 100 & / & $38(36-39)$ \\
\hline & \multirow{4}{*}{2} & \multirow[b]{2}{*}{ Abruzzo } & C. obsoletus & $2 / 2$ & 100 & 1 & $38(33-42)$ \\
\hline & & & C. scoticus & $10 / 12$ & 83.3 & I & $37(33-42)$ \\
\hline & & \multirow{2}{*}{ Lazio } & C. obsoletus & $2 / 2$ & 100 & / & $38(36-39)$ \\
\hline & & & C. scoticus & $9 / 9$ & 100 & l & $38(36-39)$ \\
\hline & \multirow{5}{*}{8} & \multirow{3}{*}{ Abruzzo } & C. obsoletus & $6 / 8$ & 75 & 1 & $42(39-45)$ \\
\hline & & & C. scoticus & $4 / 7$ & 57.1 & l & $42(39-47)$ \\
\hline & & & C. obsoletus/scoticus * & $2 / 2$ & 100 & l & $41(40-42)$ \\
\hline & & \multirow{2}{*}{ Lazio } & C. obsoletus & $6 / 6$ & 100 & 1 & $41(41-42)$ \\
\hline & & & C. scoticus & $1 / 1$ & 100 & I & $39(39-39)$ \\
\hline & \multirow{4}{*}{$4 \mathrm{MOR}$} & \multirow{2}{*}{ Abruzzo } & C. obsoletus & $3 / 5$ & 60 & 1 & $43(40-48)$ \\
\hline & & & C. scoticus & $7 / 8$ & 87.5 & / & $42(38-49)$ \\
\hline & & \multirow{2}{*}{ Lazio } & C. obsoletus & $3 / 4$ & 75 & / & $39(38-41)$ \\
\hline & & & C. scoticus & $4 / 4$ & 100 & l & $40(37-47)$ \\
\hline \multirow{11}{*}{10} & & \multirow{3}{*}{ Abruzzo } & C. obsoletus & $1 / 14$ & 7.1 & $1.7-31.9$ & $34(34-34)$ \\
\hline & & & C. scoticus & $8 / 173$ & 4.6 & $2.4-8.9$ & $41(37-46)$ \\
\hline & 4 ITA & & C. obsoletus/scoticus * & $0 / 3$ & 0 & 1 & 1 \\
\hline & & \multirow{3}{*}{ Lazio } & C. obsoletus & $3 / 39$ & 7.7 & $2.8-20.4$ & $41(38-43)$ \\
\hline & & & C. scoticus & $15 / 146$ & 10.3 & $6.4-16.3$ & $40(25-47)$ \\
\hline & & & C. obsoletus/scoticus * & $0 / 1$ & 0 & / & / \\
\hline & \multirow{5}{*}{2} & \multirow{3}{*}{ Abruzzo } & C. obsoletus & $0 / 46$ & 0 & $0.1-7.5$ & l \\
\hline & & & C. scoticus & $6 / 178$ & 3.4 & $1.6-7.2$ & $38(27-42)$ \\
\hline & & & C. obsoletus/scoticus * & $0 / 2$ & 0 & 1 & 1 \\
\hline & & \multirow{2}{*}{ Lazio } & C. obsoletus & $2 / 16$ & 12.5 & $3.8-36.4$ & $36(33-39)$ \\
\hline & & & C. scoticus & $7 / 59$ & 11.9 & $5.9-22.6$ & $39(37-40)$ \\
\hline
\end{tabular}


Table 7. Cont.

\begin{tabular}{|c|c|c|c|c|c|c|c|}
\hline Dpi & $\begin{array}{l}\text { BTV } \\
\text { Strain }\end{array}$ & Site & Species & $\begin{array}{l}\text { Number of Positive/ } \\
\text { Number of Tested }\end{array}$ & $\begin{array}{l}\text { Recovery } \\
\text { Rate \% }\end{array}$ & $\begin{array}{l}\text { Confidence } \\
\text { Intervals \% }\end{array}$ & $\begin{array}{l}\text { CT Mean } \\
\text { (Min-Max) }\end{array}$ \\
\hline \multirow{10}{*}{10} & \multirow{5}{*}{8} & \multirow{3}{*}{ Abruzzo } & C. obsoletus & $1 / 45$ & 2.2 & $0.5-11.5$ & $44(44-44)$ \\
\hline & & & C. scoticus & $3 / 63$ & 4.8 & $1.7-13.1$ & $42(39-46)$ \\
\hline & & & C. obsoletus/scoticus * & $0 / 2$ & 0 & I & 1 \\
\hline & & \multirow{2}{*}{ Lazio } & C. obsoletus & $2 / 46$ & 4.3 & $1.3-14.5$ & $42(39-44)$ \\
\hline & & & C. scoticus & $0 / 15$ & 0 & $0.2-20.6$ & 1 \\
\hline & \multirow{5}{*}{$4 \mathrm{MOR}$} & \multirow{3}{*}{ Abruzzo } & C. obsoletus & $0 / 22$ & 0 & $0.1-14.8$ & l \\
\hline & & & C. scoticus & $0 / 103$ & 0 & $0-3.5$ & I \\
\hline & & & C. obsoletus/scoticus * & $0 / 1$ & 0 & I & I \\
\hline & & \multirow{2}{*}{ Lazio } & C. obsoletus & $2 / 28$ & 7.1 & $2.2-22.8$ & $41(38-43)$ \\
\hline & & & C. scoticus & $2 / 36$ & 5.6 & $0.1-9.5$ & $40(39-41)$ \\
\hline
\end{tabular}

* species identification inconclusive.

\section{Discussion}

Vector competence is the susceptibility of the vector to infection with the pathogen and the ability of the infected vector to transmit the pathogen to a host during blood feeding. Vector competence is a key component in the vector-pathogen cycle. This study describes the vector competence of different field-collected populations of $C$. imicola and C. obsoletus and C. scoticus from different sites of Italy, after oral exposure to four bluetongue virus strains (BTV-2, BTV-4 ITA, BTV-4 MOR, and BTV-8). These strains were selected on the basis of their spread and adaptation to different episystems in Europe and the Mediterranean basin. Artificial feeding systems are valuable tools that can be used to investigate vector competence of blood feeding arthropods and have been previously utilized to investigate the vector of Culicoides midges and various BTV strains [19,37].

Midges were orally infected using the Hemotek blood feeding system through Parafilm ${ }^{\circledR}$ membrane and the cotton wool pledgets. The results obtained in this study demonstrated that Parafilm ${ }^{\circledR}$ membrane is suitable for C. imicola even though, because of its low feeding rate $(1-2.03 \%)$, a great number of midges should be used for feeding (Table 1). The same cannot be said for species of the Obsoletus complex, since even a great number of midges was not enough to achieve low feeding rates (Table 1). The reluctance of this complex to blood-feed through membrane-based systems has been previously documented [14-16]. Because of the low feeding rates achieved using the Hemotek system, vector competence comparisons were mostly made on the data obtained when midges were fed on cotton pledgets. The Hemotek membrane data were used for comparing the susceptibility of the Sardinia population of $C$. imicola to the various BTV strains only. The high feeding rate via the cotton method for both C. imicola and C. obsoletus/scoticus is in line with the results of other research groups $[18,19]$. Higher feeding rates, however, do not mean recovery rates. Although acquiring significantly lower feeding rates, the BTV recovery rates from the Sardinian population of Culicoides imicola fed through the Hemotek membrane were significantly higher than those fed on cotton pledgets. Venter [19] also found BTV recovery rates from C. imicola and C. bolitinos fed on cotton pads significantly lower than those fed through the membrane system. The authors hypothesized that the lower recovery rate was probably a consequence of the reduced blood intake (by about $30 \%$ ) of the midges fed on cotton pads. In their study, the blood meal size was calculated for each method by weighting fed (partially/full engorged not specified) and unfed midges. In this study, only the full engorged females were taken and, thus, the lower dissemination rates cannot be ascribed to a reduced blood intake but to other factors associated to the cotton wool fibers, in particular to their possible interaction with the virus, making it less accessible for midges during feeding.

Another important variable that has been shown to influence the recovery rate following artificial feeding is the virus titer of the blood meal. Paweska [38] stated that there is a strong linear correlation between the virus recovery rates from midges tested immediately after feeding and the virus titer of the blood meal. In this trial, high blood meal titers were used (ranging between $10^{5} \mathrm{TCID}_{50} / \mathrm{mL}$ and 
$\left.10^{6.54} \mathrm{TCID}_{50} / \mathrm{mL}\right)$ which are similar to the realistic viremic host titers $\left(10^{4}-10^{6} \mathrm{TCID}_{50} / \mathrm{mL}\right)[39,40]$. Nonetheless, as revealed by other studies with similar virus titers of blood meals and corroborated in the current study, few midges were still negative when tested immediately after feeding [22,24].

The vector competence of the Culicoides populations involved in this trial was determined by testing the heads of midges using serogroup-specific and serotype-specific RT-PCRs after an incubation period of ten days. Positive heads implicated a potential competence for BTV transmission due to the absence of a salivary gland barrier to BTV in Culicoides [17,35]. As expected, the serogroup-specific RT-PCR was more sensitive than the type-specific RT-PCR. However, in Sardinia, in the farm where field C. imicola midges were trapped for BTV-8 competence studies, BTV-4 was circulating at the time of collection. Thus, to avoid false positive results which could be derived from the use of serogroup-specific RT-PCR in testing BTV-4 positive midges, the type-specific RT-PCR was included in this study. Its results were used in all vector competence comparisons, which included BTV- 8 and the Sardinian population of $C$. imicola. For the remainder of the recovery rate comparisons, the results of the serotype-specific RT-PCR assay were used.

Another important element to take into consideration for the evaluation of the vector competency is the RT-PCR Ct value at 0 and $10 \mathrm{dpi}$. Ct values at $10 \mathrm{dpi}$ lower than those found at 0 dpi may imply virus replication in the salivary glands [25] and, ultimately, a role for oral transmission. In analyzing the recovery rate data, more or less conservative approaches have been evaluated based on the RT-PCR cut off levels. Because of the great variations of the RT-PCR results and the Ct values observed, to have solid competence data and avoid possible overestimation, a more conservative approach was taken, and only those cases where the RT-PCR Ct values at $10 \mathrm{dpi}$ were lower than the $\mathrm{Ct}$ mean values observed at 0 dpi were included in the analysis. Apart from the Abruzzo population of $C$. obsoletus/ C. scoticus, which was refractory to BTV-4 MOR infection, all orally infected vector populations tested in this study were able to sustain the BTV strain replication, even that of BTV-4 MOR, a strain which has never been reported in Italy. Unfortunately, the rather low proportion of infected midges obtained after the artificial feeding precluded some competence evaluations and reduced the statistical power of the comparisons.

Overall, BTV recovery rates from C. imicola were significantly higher than those recovered from species of the Obsoletus complex. In other words, this study indicated a probably higher susceptibility of C. imicola to the selected BTV strains in comparison to the Obsoletus complex populations. To the best of our knowledge, this is the first time that it was possible to compare the competences of either Culicoides species. Considering the Obsoletus complex populations, C. obsoletus and C. scoticus were present with a predominance of $C$. scoticus in either populations. Both species were susceptible to all BTV without any significant difference of recovery rates. Differences of recovery rate among Culicoides populations could definitely influence the distribution of BTV strains [5]. Regardless, it must be taken into account that a low susceptibility does not necessarily imply a low vector capacity. Vector competence is just a component of vector capacity, so other elements (high abundance, wide distribution, high biting rate, etc.) are important for virus spread. A low susceptibility could be compensated for by the abundance and wide distribution, as in the case of $C$. imicola and species of the Obsoletus complex in Italy. Susceptibility to infection with BTV can vary in Culicoides at species and populations levels [5,37]. It is difficult to understand the cause of the variation in recovery rates between different populations, especially of the same species, since interactions between virus and midge are very complex and poorly investigated. Vector competence could be influenced by hereditary and environmental factors, including the composition of gut microbiota [41]. It has been observed that a blood meal on its own alters the composition/abundance of gut microbiota and influences the expression of antimicrobial peptides [42]. Further studies about transcriptomic analysis and virome and microbiome composition of the Italian populations of Culicoides analyzed in this work might allow better understanding these complex and dynamic mechanisms of Culicoides infection and vector competence. 
Significant differences were also observed between the BTV recovery rates from the Culicoides populations tested in this trial. Particularly interesting are the results obtained in the Sardinian population of $C$. imicola after feeding through the Hemotek membrane system. Sardinia has represented and represents the main entrance for most of the Italian BTV incursions. From the Sardinian midge population, the recovery rates of BTV-2, BTV-4 ITAm and BTV-8 were higher than the rate observed for BTV-4 MOR. The higher competence shown by the Sardinian population of $C$. imicola for these 3 BTV strains could explain their successful spread in the island during the BTV-2, BTV-8, and BTV-4 incursions occurring in 2000-2001, 2009, and 2016-2017, respectively [10]. In the same way, it could also be one of the reasons for the possible unsuccessful incursion attempts of BTV-4 MOR. Feeding Culicoides midges on cotton wool also evidenced differences in the BTV-4ITA recovery rates from the Sardinian and Abruzzo Culicoides populations. The recovery rate from the Sardinia C. imicola population (5\%, 95\% CI 2.99-8.43\%) was significantly higher than that obtained from the C. obsoletus/scoticus of Abruzzo $(0.53 \%, 95 \% \mathrm{CI} 0.13-2.88 \% \%)$. This different susceptibility might have contributed to the different impact that the 2016-2017 BTV-4ITA incursion had on the livestock of the two regions. More than 70,000 animals were affected in Sardinia, with an infectious rate of $13.62 \%$ (95\%CI: 13.53-13.72\%), while the affected animals in Abruzzo were only 31, with an infectious rate of 2.84\% (95\%CI: $2.01-4.01 \%$ ).

Even though numerous studies on vector competence have been performed, results are in most cases not comparable because of the variety of the infection protocols used. Culicoides imicola has been proven to be susceptible to several BTV serotypes, including BTV-2 [43], BTV-4 [20,43], and BTV-8 [22,23]. Species of the Obsoletus complex, although less investigated, have been found to be susceptible to serotypes $1,4,8$, and $9[17,18]$. The authors of [43] achieved low recovery rates when C. imicola midges were fed with BTV-2 (0.5\%), BTV-4 (0.2\%), and BTV-8 (0\%) infected blood through membrane. Relatively low recovery rates of either BTV-8 ( $<1 \%$ for both European and South African strains) or BTV-4 (1.9\%), using the virus isolation technique, were also achieved from C. imicola fed on 1-day-old chicken membrane $[20,22,23]$. There were also cases where some BTV strains (BTV-4 and BTV-2) were not even recovered from midges [22]. Paslaru [17] demonstrated the competence of the Obsoletus complex for BTV-8 and BTV-4, obtaining a dissemination rate of $2 \%$ for both serotypes. Virus detection was performed by RT-PCR on heads as in our study, but the feeding method and the length and temperature of incubation period were not the same. Thus, also in this case, the variability of important parameters makes it difficult to compare the results.

\section{Conclusions}

This study has provided some important data on the vector competence of different $C$. imicola and C. obsoletus/scoticus populations in Italy in relation to four BTV strains. The results obtained prove that, except for the Abruzzo population of C. obsoletus/C. scoticus, which was refractory to BTV-4 MOR infection, all the investigated populations were susceptible to the BTV strains used in the trial, showing, in some instances, significant differences in recovery rates depending on midge population and/or BTV strain. Considering the wide distribution of C. obsoletus and C. scoticus in Europe, these findings provided evidence that strains like BTV-2 and BTV-4 MOR could have spread all over Europe. Their dissemination failure might have been a consequence of successful vaccination programs and the correct application of restriction measures. Their success was a consequence of contemporaneous appliance of both vaccination of susceptible animals and restricting movement of viremic animals between BT-affected and BT-free zones. The BTV-2 and BTV-4 vaccination campaigns used either modified live vaccines (BTV-2 and BTV-4 outbreaks in Balearic Islands and Corsica in 2000 and 2001, Italy 2003-2004) or inactivated vaccines (BTV-4 outbreaks in Southwest Spain in 2004 and 2005). Both campaigns targeted all susceptible ruminant species, achieved a high degree of herd immunity, and included extensive areas surrounding any active BT outbreaks [44].

Author Contributions: Conceptualization, M.G. and G.S. (Giovanni Savini); Investigation, M.D.A., F.D.N., V.F., G.M., M.Q., A.S., O.P., M.P., A.L., F.P., and L.T.; Data curation, V.F. and L.T.; Formal analysis, V.F.; 
Project administration, P.C., V.F., G.M., and C.V.; Supervision, C.D.L., G.F., G.M., and G.S. (Giuseppe Satta); Writing—original draft, V.F.; Writing—review and editing, M.G. and G.S. (Giovanni Savini).

Funding: This project has received funding from the European Union's Seventh Framework Programme for research, technological development and demonstration under grant agreement no 291815.

Acknowledgments: The authors wish to thank Lara Harrup and The Pirbright Institute Orbivirus Reference Collection for the provision of viral strains.

Conflicts of Interest: The authors declare no conflict of interest. The funders had no role in the design of the study; in the collection, analyses, or interpretation of data; in the writing of the manuscript, or in the decision to publish the results.

\section{References}

1. Simón, F.; González-Miguel, J.; Diosdado, A.; Gómez, P.J.; Morchón, R.; Kartashev, V. The complexity of zoonotic filariasis episystem and its consequences: A multidisciplinary view. BioMed. Res. Int. 2017, 2017, 6436130. [CrossRef] [PubMed]

2. Goffredo, M.; Conte, A.; Cocciolito, R.; Meiswinkel, R. Distribuzione e abbondanza di Culicoides imicola in Italia. Vet. Ital. 2003, 39, 22-32.

3. Goffredo, M.; Catalani, M.; Federici, V.; Portanti, O.; Marini, V.; Mancini, G.; Quaglia, M.; Santilli, A.; Teodori, L.; Savini, G. Vector species of Culicoides midges implicated in the 2012-2014 Bluetongue epidemics in Italy. Vet. Ital. 2015, 51, 131-138. [CrossRef]

4. Goffredo, M.; Meiswinkel, R.; Federici, V.; Di Nicola, F.; Mancini, G.; Ippoliti, C.; Di Lorenzo, A.; Quaglia, M.; Santilli, A.; Conte, A.; et al. The "Culicoides obsoletus group" in Italy: Relative abundance, geographic range, and role as vector for Bluetongue virus. Vet. Ital. 2016, 52, 235-241. [CrossRef] [PubMed]

5. Tabachnick, W.J. Culicoides variipennis and bluetongue-virus epidemiology in the United States. Annu. Rev. Entomol. 1996, 41, 23-43. [CrossRef]

6. Conte, A.; Goffredo, M.; Ippoliti, C.; Meiswinkel, R. Influence of biotic and abiotic factors on the distribution and abundance of Culicoides imicola and the Obsoletus Complex in Italy. Vet. Parasitol. 2007, 150, 333-344. [CrossRef]

7. Calistri, P.; Giovannini, A.; Conte, A.; Nannini, D.A.O.; Santucci, U.; Patta, C.; Rolesu, S.; Caporale, V. Bluetongue in Italy: Part I. Vet. Ital. 2004, 40, 243-251.

8. Foxi, C.; Delrio, G.; Falchi, G.; Marche, M.G.; Satta, G.; Ruiu, L. Role of different Culicoides vectors (Diptera: Ceratopogonidae) in bluetongue virus transmission and overwintering in Sardinia (Italy). Parasites Vectors 2016, 9, 440. [CrossRef]

9. Savini, G.; Goffredo, M.; Monaco, F.; Di Gennaro, A.; Cafiero, M.A.; Baldi, L.; De Santis, P.; Meiswinkel, R.; Caporale, V. Bluetongue virus isolations from midges belonging to the Obsoletus complex (Culicoides, Diptera: Ceratopogonidae) in Italy. Vet. Rec. 2005, 157, 133. [CrossRef]

10. EFSA Panel on Animal Health and Welfare (AHAW); More, S.; Bicout, D.; Bøtner, A.; Butterworth, A.; Depner, K.; Edwards, S.; Garin-Bastuji, B.; Good, M.; Gortázar, S.C.; et al. Assessment of listing and categorisation of animal diseases within the framework of the Animal Health Law (Regulation (EU) No 2016/429): Bluetongue. Efsa J. 2017, 15, e04957. [CrossRef]

11. Lorusso, A.; Guercio, A.; Purpari, G.; Cammà, C.; Calistri, P.; D’Alterio, N.; Hammami, S.; Sghaier, S.; Savini, G. Bluetongue virus serotype 3 in Western Sicily, November 2017. Vet. Ital. 2017, 53, 273-275. [PubMed]

12. Lhor, Y.; Kyriaki, N.; Khayli, M.; Bouslikhane, M.; Fassi Fihri, O.; El Harrak, M. Bluetongue in Morocco 2004 to 2015: An overview. J. Infect. Dis. Epidemiol. 2017, 3, 23. [CrossRef]

13. Lhor, Y.; Khayli, M.; Bouslikhane, M.; El Harrak, M.; Fihri, O.F. Spatial and seasonal distribution of Culicoides species in Morocco in relation to the transmission of Bluetongue Viruses. Br. J. Virol. 2015, 2, 88-95. [CrossRef]

14. Goffredo, M.; Romeo, G.; Monaco, F.; Di Gennaro, A.; Savini, G. Laboratory survival and blood feeding response of wild-caught Culicoides obsoletus Complex (Diptera: Ceratopogonidae) through natural and artificial membranes. Vet. Ital. 2004, 40, 282-285.

15. Jennings, D.M.; Mellor, P.S. The vector potential of British Culicoides species for bluetongue virus. Vet. Microbiol. 1988, 17, 1-10. [CrossRef] 
16. Mellor, P.S. Culicoides as potential orbivirus vectors in Europe. In Bluetongue, African Horse Sickness, and Related Orbiviruses: Proceedings of the Second International Symposium; CRC Press: Boca Raton, FL, USA, 1992; pp. 278-283.

17. Paslaru, A.I.; Mathis, A.; Torgerson, P.; Veronesi, E. Vector competence of pre-alpine Culicoides (Diptera: Ceratopogonidae) for bluetongue virus serotypes 1, 4 and 8. Parasites Vectors 2018, 11, 466. [CrossRef]

18. Carpenter, S.; McArthur, C.; Selby, R.; Ward, R.; Nolan, D.V.; Luntz, A.J.; Dallas, J.F.; Tripet, F.; Mellor, P.S. Experimental infection studies of UK Culicoides species midges with bluetongue virus serotypes 8 and 9 . Vet. Rec. 2008, 163, 589. [CrossRef]

19. Venter, G.J.; Paweska, J.T.; Lunt, H.; Mellor, P.S.; Carpenter, S. An alternative method of blood-feeding Culicoides imicola and other haematophagous Culicoides species for vector competence studies. Vet. Parasitol. 2005, 131, 331-335. [CrossRef]

20. Venter, G.J.; Paweska, J.T.; Van Dijk, A.A.; Mellor, P.S.; Tabachnick, W.J. Vector competence of Culicoides bolitinos and C. imicola for South African bluetongue virus serotypes 1, 3 and 4. Med. Vet. Entomol. 1998, 12, 378-385. [CrossRef]

21. Venter, G.J.; Gerdes, G.H.; Mellor, P.S.; Paweska, J.T. Transmission potential of South African Culicoides species for live-attenuated bluetongue virus. Vet. Ital. 2004, 40, 199.

22. Venter, G.J.; Mellor, P.S.; Paweska, J.T. Oral susceptibility of South African stock-associated Culicoides species to bluetongue virus. Med. Vet. Entomol. 2006, 20, 329-334. [CrossRef] [PubMed]

23. Venter, G.J.; Wright, I.M.; Del Rio, R.; Lucientes, J.; Miranda, M.A. The susceptibility of Culicoides imicola and other South African livestock-associated Culicoides species to infection with bluetongue virus serotype 8 . Med. Vet. Entomol. 2011, 25, 320-326. [CrossRef] [PubMed]

24. Paweska, J.T.; Prinsloo, S.; Venter, G.J. Oral susceptibility of South African Culicoides species to live-attenuated serotype-specific vaccine strains of African horse sickness virus (AHSV). Med. Vet. Entomol. 2003, 17, $436-447$. [CrossRef] [PubMed]

25. Veronesi, E.; Antony, F.; Gubbins, S.; Golding, N.; Blackwell, A.; Mertens, P.P.; Brownlie, J.; Darpel, K.E.; Mellor, P.S.; Carpenter, S. Measurement of the infection and dissemination of bluetongue virus in Culicoides biting midges using a semi-quantitative rt-PCR assay and isolation of infectious virus. PLoS ONE 2013, 8, e70800. [CrossRef]

26. Veronesi, E.; Henstock, M.; Gubbins, S.; Batten, C.; Manley, R.; Barber, J.; Hoffmann, B.; Beer, M.; Attoui, H.; Mertens, P.P.; et al. Implicating Culicoides biting midges as vectors of Schmallenberg virus using semi-quantitative RT-PCR. PLoS ONE 2013, 8, e57747. [CrossRef]

27. Barber, J.; Harrup, L.E.; Silk, R.; Veronesi, E.; Gubbins, S.; Bachanek-Bankowska, K.; Carpenter, S. Blood-feeding, susceptibility to infection with Schmallenberg virus and phylogenetics of Culicoides (Diptera: Ceratopogonidae) from the United Kingdom. Parasites Vectors 2018, 11, 116. [CrossRef]

28. Reed, L.J.; Muench, H. A simple method of estimating fifty per cent endpoints. Am. J. Epidemiol. 1938, 27, 493-497. [CrossRef]

29. Delécolle, J.C. Nouvelle contribution à l'étude systématique et iconographique des espèces du genre Culicoides (Diptera: Ceratopogonidae) du Nord-Est de la France. Ph.D. Thesis, Université Louis Pasteur de Strasbourg, Strasbourg, France, 1985.

30. Campbell, J.A.; Pelham-Clinton, E.C. X.-A taxonomic review of the British species of Culicoides Latreille (Diptera: Ceratopogonidae). Proc. R. Soc. Edinb. Sect. B Biol. Sci. 1960, 67, 181-302. [CrossRef]

31. Goffredo, M.; Meiswinkel, R. Entomological surveillance of bluetongue in Italy: Methods of capture, catch analysis and identification of Culicoides biting midges. Vet. Ital. 2004, 40, 260-265.

32. Gomulski, L.M.; Meiswinkel, R.; Delécolle, J.C.; Goffredo, M.; Gasperi, G. Phylogenetic relationships of the subgenus Avaritia Fox, 1955 including Culicoides obsoletus (Diptera, Ceratopogonidae) in Italy based on internal transcribed spacer 2 ribosomal DNA sequences. Syst. Entomol. 2005, 30, 619-631. [CrossRef]

33. Sarvašová, A.; Goffredo, M.; Sopoliga, I.; Savini, G.; Kočišová, A. Culicoides midges (Diptera: Ceratopogonidae) as vectors of orbiviruses in Slovakia. Vet. Ital. 2014, 50, 203-212. [CrossRef] [PubMed]

34. Hofmann, M.A.; Griot, C.; Renzullo, S.; Mader, M.; Chaignat, V.; Worwa, G.; Thuer, B. Genetic characterization of toggenburg orbivirus, a new bluetongue virus, from goats, Switzerland. Emerg. Infect. Dis. 2008, 14, 1855-1861. [CrossRef] [PubMed] 
35. Fu, H.; Leake, C.J.; Mertens, P.P.C.; Mellor, P.S. The barriers to bluetongue virus infection, dissemination and transmission in the vector, Culicoides variipennis (Diptera: Ceratopogonidae). Arch. Virol. 1999, 144, 747-761. [CrossRef] [PubMed]

36. Sivia, D.S. Data Analysis: A Bayesian Tutorial; Clarendon Press: Oxford, UK, 1996; pp. 106-110.

37. Carpenter, S.; Lunt, H.L.; Arav, D.; Venter, G.J.; Mellor, P.S. Oral susceptibility to bluetongue virus of Culicoides (Diptera: Ceratopogonidae) from the United Kingdom. J. Med. Entomol. 2006, 43, 73-78. [CrossRef]

38. Paweska, J.T.; Venter, G.J.; Hamblin, C. A comparison of the susceptibility of Culicoides imicola and C. bolitinos to oral infection with eight serotypes of epizootic haemorrhagic disease virus. Med. Vet. Entomol. 2005, 19, 200-207. [CrossRef]

39. Savini, G.; Monaco, F.; Facchinei, A.; Pinoni, C.; Salucci, S.; Cofini, F.; Di Ventura, M. Field vaccination of sheep with bivalent modified-live vaccine against bluetongue virus serotypes 2 and 9: Effect on milk production. Vet. Ital. 2004, 40, 627-630.

40. Savini, G.; Monaco, F.; Citarella, R.; Calzetta, G.A.O.; Panichi, G.; Ruiu, A.; Caporale, V. Monovalent modifiedlive vaccine against bluetongue virus serotype 2: Immunity studies in cows. Vet. Ital. 2004, 40, 664-667.

41. Mills, M.K.; Michel, K.; Pfannenstiel, R.S.; Ruder, M.G.; Veronesi, E.; Nayduch, D. Culicoides-virus interactions: Infection barriers and possible factors underlying vector competence. Curr. Opin. Insect. Sci. 2017, 22, 7-15. [CrossRef]

42. Nayduch, D.; Erram, D.; Lee, M.B.; Zurek, L.; Saski, C.A. Impact of the blood meal on humoral immunity and microbiota in the gut of female Culicoides sonorensis. Vet. Ital. 2015, 51, 385-392. [CrossRef]

43. Del Rio Lopez, R.; Miranda, M.A.; Paredes-Esquivel, C.; Lucientes, J.; Calvete, C.; Estrada, R.; Venter, G.J. Recovery rates of bluetongue virus serotypes 1, 2, 4 and 8 Spanish strains from orally infected Culicoides imicola in South Africa. Med. Vet. Entomol. 2012, 26, 162-167. [CrossRef]

44. Savini, G.; MacLachlan, N.J.; Sanchez-Vizcaino, J.M.; Zientara, S. Vaccines against bluetongue in Europe. Comp. Immunol. Microbiol. Infect. Dis. 2008, 31, 101-120. [CrossRef] [PubMed]

(C) 2019 by the authors. Licensee MDPI, Basel, Switzerland. This article is an open access article distributed under the terms and conditions of the Creative Commons Attribution (CC BY) license (http://creativecommons.org/licenses/by/4.0/). 